\title{
Characterisation of Cascaded Raman-Assisted Fibre Optical Parametric Amplifiers using WDM QPSK Signals
}

\author{
Alexey Redyuk ${ }^{1,2}$, Marc F.C. Stephens ${ }^{3}$, and Nick J. Doran ${ }^{3}$ \\ ${ }^{I}$ Institute of Computational Technologies, Novosibirsk, 6 Acad. Lavrentiev avenue, 630090, Russia, redyuk@ict.sbras.ru \\ ${ }^{2}$ Novosibirsk State University, Novosibirsk, 2 Pirogova street, 630090, Russia \\ 3Aston Institute of Photonic Technologies, Aston University, Aston Triangle, Birmingham B4 7ET, UK, m.stephens@aston.ac.uk
}

\begin{abstract}
We report the first WDM numerical characterisation of crosstalk growth in cascaded Raman-Assisted Fibre Optical Parametric Amplifiers (RA-FOPAs). A cascade of ten RA-FOPAs results in $\sim 13 \mathrm{~dB}$ lower crosstalk than the equivalent cascade of conventional FOPAs.

OCIS codes: (190.4970) Parametric oscillators and amplifiers; (190.4380) Nonlinear optics, four-wave mixing.
\end{abstract}

\section{Introduction}

A logical way of addressing worldwide data-transport capacity demands is to provide amplification for optical signals extending beyond traditional $\mathrm{C} / \mathrm{L}$ bands [1]. The Raman-Assisted Fibre Optical Parametric Amplifier (RA-FOPA) has been shown to be a promising candidate for achieving this [2-4]. The RA-FOPA combines numerous useful properties of discrete Raman (low crosstalk, non-resonant gain) with those of conventional FOPAs (high gain coefficient, non-resonant gain) to offer a tuneable gain-region and potentially high discrete gain together with a potential for reduced four-wave mixing (FWM) WDM crosstalk over the standard FOPA [3,4].

In our previous work [4] we reported that the RA-FOPA crosstalk can be minimised by combining a short HNLF length with maximum available Raman pump power and a parametric pump power subsequently-adjusted to achieve the required operating net gain level. In this paper, we extend our previous work by examining the evolution of crosstalk growth in a cascaded series of both RA-FOPAs and conventional FOPAs carrying WDM QPSK signals. In this way we can ascertain their respective suitability for long distance transmission. We find that after a cascade of ten amplifications, the worst-case crosstalk growth arising from transmission of $10 x 58 \mathrm{~Gb} / \mathrm{s}$ signals is up to $13 \mathrm{~dB}$ lower in the optimised RA-FOPA.

\section{Mathematical model and methodology}

The RA-FOPA experimental and simulated schematic is shown in Figure 1(a). For a dedicated comparison with our previous results [3], the input signals comprised of ten 100GHz-spaced NRZ-QPSK modulated channels ranging from 193.5 to $194.4 \mathrm{THz}$ and multiplexed together using a $70 \mathrm{GHz}$-wide arrayed waveguide grating (WDM1). The QPSK modulation data was derived from two decorrelated $2^{12}$ pseudo-random binary (PRBS) bit sequences at a symbol rate of $29 \mathrm{Gbaud} / \mathrm{s}$. A $100 \mathrm{kHz}$ parametric pump (PP) laser was phase modulated with a $3 \mathrm{~Gb} / \mathrm{s}$ PRBS pattern to reduce stimulated Brillouin scattering and optically amplified.

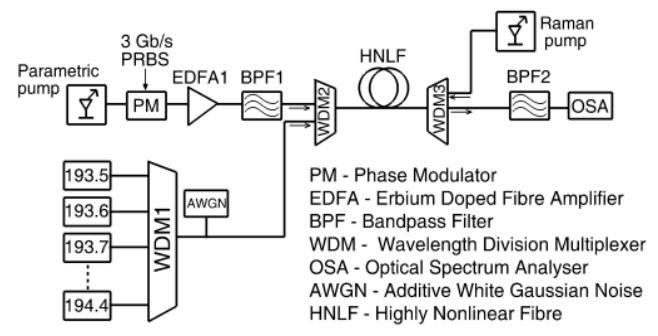

(a)

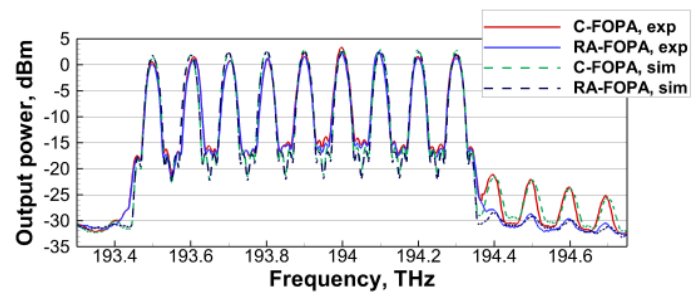

(b)

Fig. 1. (a) Scheme of simulation for Raman-assisted FOPA (b) Output spectra of the RA-FOPA and C-FOPA at $12.5 \mathrm{GHz}$ resolution bandwidth for $-12 \mathrm{dBm}$ per-signal input power and $20 \mathrm{~dB}$ average net gain.

The PP was bandpass filtered (BPF1) to remove amplified spontaneous emission (ASE) before combination with the signals using filter WDM2. The combined pump/signals were transmitted through $0.2 \mathrm{~km}$ of HNLF with the following properties: fiber loss $0.8 \mathrm{~dB} / \mathrm{km}$; zero dispersion wavelength $1564.3 \mathrm{~nm}$; dispersion slope $0.084 \mathrm{psnm}^{-2} \mathrm{~km}^{-1}$; and nonlinear coefficient $8.2\left(\mathrm{~W} \mathrm{~km}^{-1}\right.$. Per-signal input power to the HNLF was fixed at a relatively high level of $-10 \mathrm{dBm}$ in order to generate significant FWM crosstalk under C-FOPA operation. For RA-FOPA operation, the HNLF was additionally backward-pumped using a continuous-wave (CW) Raman pump (RP) of maximum power $37 \mathrm{dBm}(5 \mathrm{~W})$ at $1455 \mathrm{~nm}$. The power and wavelength of the PP and power of the RP were adjustable variables to 
achieve the required net-gain for all signals as either a) a conventional C-FOPA (no Raman) or b) a hybrid RA-FOPA. All pumps and signals were simulated as polarisation-aligned for maximum signal gain and worst case crosstalk growth through the HNLF.

The RA-FOPA modelling process consisted of two stages: bidirectional power analysis followed by time-domain field analysis [5]. In the first stage, the interaction between the signals, co-propagating parametric pump and counter propagating Raman pump was determined using coupled balanced equations for the time-averaged power of the Raman pump and the total average power of the WDM signals and parametric pump. The approximate solution of coupled balanced equations was obtained iteratively using the fourth-order Runge-Kutta method. For the second stage, the resultant power distributions were substituted along the fiber length into the nonlinear Schrödinger equation (NLSE) containing a delayed Raman response term and solved via the standard split-step Fourier method.

Experimental and simulated output spectra of the C-FOPA and RA-FOPA are shown in Figure 1(b) for the representative conditions of $20 \mathrm{~dB}$ net-gain and $-12 \mathrm{dBm}$ input power per signal. It can be seen that there is close agreement of signal power, spectral flatness and crosstalk distribution for both schemes, providing confidence in the simulation predictions.

\section{Results and discussion}

The generation of both parametric and Raman gain in a single length of HNLF (RA-FOPA) offers a useful advantage over a hybrid amplifier consisting of both a discrete C-FOPA and a discrete Raman amplifier (or vice versa). This is because the peak of the Raman gain in the RA-FOPA can be tuned to coincide (and thus provide gain) to both the WDM signals and the parametric pump. The latter is important because it provides additional signal amplification due to the parametric process. To understand and illustrate this phenomenon, three $20 \mathrm{~dB}$ netgain scenarios were compared for the same $0.2 \mathrm{~km}$ HNLF: a) C-FOPA with $33.4 \mathrm{dBm}$ PP b) RA-FOPA1 with $32.1 \mathrm{dBm}$ PP \& $32 \mathrm{dBm}$ RP and c) RA-FOPA2 with $28.5 \mathrm{dBm}$ PP \& 37dBm RP. Figure 2(a) shows the evolution of the 194.4THz signal and PP power along the length of HNLF whilst all ten WDM signals are amplified. Important differences in signal profile between the C-FOPA and RA-FOPA are evident. For the RA-FOPA, the rate of change of signal gain increases along the HNLF but is approximately constant in the C-FOPA. This is a direct result of the counter-propagated RP and consequential amplification of the PP. It should also be noted that the C-FOPA signal gain saturates under these conditions but substantial margin remains for the RA-FOPA.

A key conclusion of the above is that under a fixed gain condition the RA-FOPA can be operated with lower parametric pump power than the equivalent C-FOPA which results in reduced FWM crosstalk [4]. To understand the behavior, the signal to crosstalk power ratio (S-to-X) was simulated at different wavelengths across the band. This was calculated by running two simulations per measurement, both with and without the channel under test present. When not present, the input power of the remaining nine channels was increased to keep total signal HNLF launch power constant.

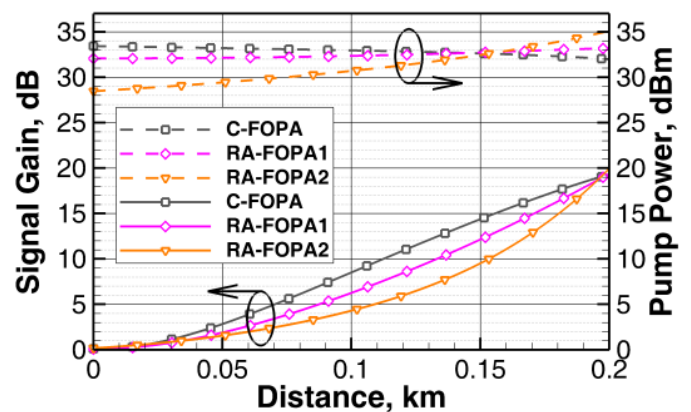

(a)

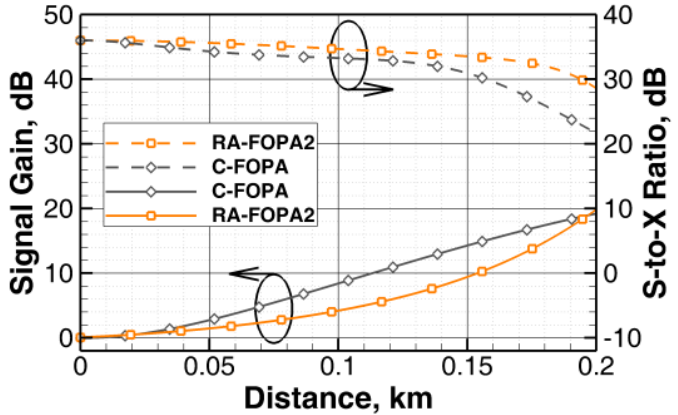

(b)

Fig. 2. (a) Signal gain and $P P$ power along the HNLF for a C-FOPA ( $P P=33.4 \mathrm{dBm})$, RA-FOPA1 $(\mathrm{PP}=32.1 \mathrm{dBm}, \mathrm{RP}=32 \mathrm{dBm})$ and RA-FOPA2 ( $\mathrm{PP}=28.5 \mathrm{dBm}, \mathrm{RP}=37 \mathrm{dBm}$ ) (b) Signal gain and $\mathrm{S}$-to-X ratio along the HNLF for a C-FOPA and RA-FOPA2.

Figure 2(b) shows the dynamics of the signal gain and S-to-X ratio at 194.4THz (largest WDM crosstalk product for this system - see Fig. 1(b)) along the HNLF for 20dB net gain. It can be seen that there is an inflection point in the S-to-X ratio profile. This occurs where the crosstalk power begins to dominate the ASE floor. Note that in the case of the C-FOPA, this point always occurs after a shorter transmitted distance through the amplifier than the equivalent RA-FOPA. This results in a 7dB difference of the S-to-X ratio between C-FOPA and RA-FOPA2 for the $0.2 \mathrm{~km}$ lengths of HNLF on a single amplification. 
To examine the crosstalk growth in a cascaded series of both types of amplifier, simulations were performed under a standardized 20dB net-gain condition for the C-FOPA, RA-FOPA1 and RA-FOPA2. To minimise simulation run time, and to keep the results relevant to the amplifier performance only, the cascade chain was varied from 1 to 10 consecutive stages, with each stage consisting of an optical attenuator (rather than transmission fibre) and a dispersion module followed by the amplifier under analysis. The attenuator loss was set to $20 \mathrm{~dB}$, and the dispersion of the module $1600 \mathrm{ps}^{-\mathrm{nm}^{-1}}$ (i.e. equivalent to $100 \mathrm{~km}$ of standard single-mode fiber). Figure 3(a) shows the S-to-X ratio profile for the worst-case $194.4 \mathrm{THz}$ signal as it evolves through the HNLF after the $1^{\text {st }}, 5^{\text {th }}$ and $10^{\text {th }}$ cascade. Only the curves for the C-FOPA and RA-FOPA are displayed for clarity. It can be seen that the first amplifier in the chain shows by far the largest divergence in crosstalk growth through the HNLF when comparing the C-FOPA and RA-FOPA2 evolution. This large divergence between the two is not replicated in later amplifications because contribution of crosstalk components due to four-wave mixing of adjacent WDM signals to crosstalk growth decreases as the number of amplification stage increases.

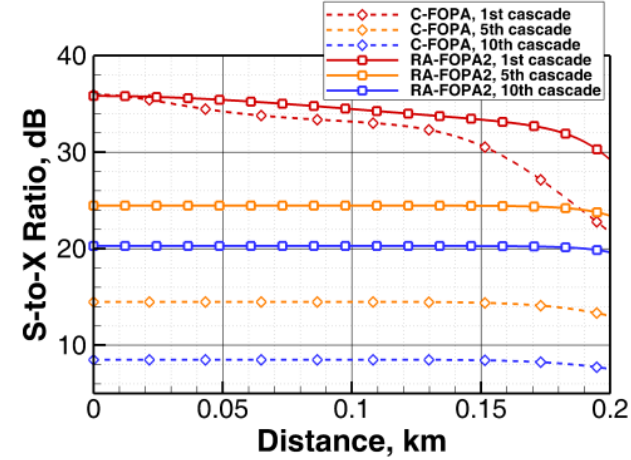

(a)

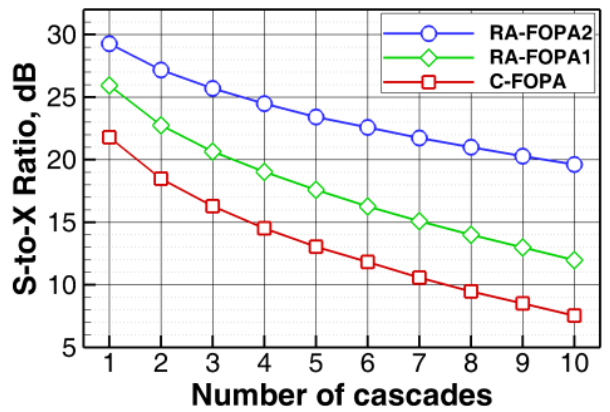

(b)

Fig. 3. (a) S-to-X ratio along the HNLF for cascaded C-FOPA and RA-FOPA2 with different number of cascades and $20 \mathrm{~dB}$ net-gain (b) Output S-to-X ratio vs number of cascades for $20 \mathrm{~dB}$ net gain.

The evolution of output S-to-X ratio after the Nth amplifier in the chain (where $\mathrm{N}=1-10$ ) was subsequently calculated and plotted in Figure 3(b), again for the signal at 194.4THz. It can be seen that the S-to-X ratio decreases as expected as the number of cascades increases (FWM power is proportional to total propagating length). Overall it can be seen that there is an increasing benefit in using the RA-FOPA2 over both the C-FOPA and RA-FOPA1 as the number of cascades increases, rising from $\sim 7 \mathrm{~dB}$ after one cascade to $\sim 13 \mathrm{~dB}$ after ten cascades. As a result, we believe that hybrid RA-FOPA amplification using a high proportion of Raman pumping shows promising cascadability for short-haul transmission links of $\sim 1000 \mathrm{~km}$, and would benefit from further study.

\section{Conclusions}

We have performed numerical simulations characterising the four wave mixing crosstalk growth in cascaded chains of both conventional FOPAs and RA-FOPAs for the first time. Under our simulated conditions (which have been shown to agree well with experiment under a single amplification), the rate of growth of crosstalk is shown to decrease with number of cascades for both the C-FOPA and RA-FOPA variants. In particular, when employing the highest proportion of Raman power in the RA-FOPA, the rate of growth of crosstalk can be slower than that of both the C-FOPA and a low-proportion Raman RA-FOPA. In this case, after ten cascades of 10x58Gb/s QPSK signals, the RA-FOPA was found to have $\sim 13 \mathrm{~dB}$ lower worst-case crosstalk than the conventional FOPA of the same gain.

\section{Acknowledgments}

This work was partially funded by the UK EPSRC grant EP/J009709/2 and the Ministry of Education and Science of the Russian Federation (no. RFMEFI57814X0029).

\section{References}

1. R.-J. Essiambre et al.,“Capacity Limits of Optical Fiber Networks,” J. Lightwave Technol. 28, 662-701 (2010).

2. X. Guo, X. Fu, and C. Shu, "Gain-Saturated Spectral Characteristic in a Raman-Assisted Fiber Optical Parametric Amplifier," Opt. Lett. Vol. 39, 3658-3661 (2014).

3. M. F. C. Stephens et al., "Improved WDM performance of a fibre optical parametric amplifier using Raman assisted pumping," Opt. Express 23, 902-911 (2015).

4. A. Redyuk et al., "Suppression of WDM four-wave mixing crosstalk in fibre optic parametric amplifier using Raman-assisted pumping," Opt. Express 23, 27240-27249 (2015).

5. G. P. Agrawal, Nonlinear Fiber Optics (Academic Press, 2001). 